\title{
COMPARISON OF ANTIBACTERIAL AND ANTIFUNGAL EFFECTS OF DIFFERENT VARIETIES OF HONEY AND PROPOLIS SAMPLES
}

\author{
S. Kolayli ${ }^{\mathrm{a}}$, I. PalabiyiK ${ }^{\mathrm{b}}$, D.S. Atik ${ }^{\mathrm{b}}$, M. Keskin ${ }^{*}$, A. Bozdeveci ${ }^{\mathrm{d}}$ and S.A. Karaoglu ${ }^{\mathrm{d}}$ \\ ${ }^{a}$ Department of Chemistry, Karadeniz Technical University, Trabzon. Turkey \\ ${ }^{b}$ Department of Food Engineering, Tekirdağ Namık Kemal University, Tekirdağ. Turkey \\ ${ }^{c}$ Vocational School of Health Services, Bilecik Şeyh Edebali University, Bilecik. Turkey \\ ${ }^{\mathrm{d}}$ Department of Biology, Recep Tayyip Erdoğan University, Rize. Turkey
}

(Received: 22 May 2020; accepted: 6 July 2020)

\begin{abstract}
Honey is the most important bee product. There are many secondary metabolites, carbohydrates, enzymes, and vitamins in honey, thus, honey has antimicrobial activity. In this study, in vitro antimicrobial activity of forty-two honey and eight propolis ethanolic extracts (PEE) were investigated against 16 microorganisms. Total phenolic content ranged between 20.00-124.10 mg GAE/100 g and 103-232 mg GAE/g for honey and raw propolis samples, respectively. Pine and oak honeydew honeys had higher antimicrobial activity than four different grades of Manuka Honeys up to $18 \mathrm{~mm}$ minimum inhibition zone diameters. The ethanolic propolis extracts showed much higher antimicrobial activity than the honey samples. Fungi species were inhibited by the propolis samples. Helicobacter pylorii (H. pylorii) was the most sensitive, whereas Streptococcus agalactiae was the most resistant bacteria among the studied microorganisms. Brazilian and Zonguldak propolis had the closest antimicrobial activity to ampicillin, streptomycin, and fluconazole. It can be concluded that both honey and propolis could be used in preservative and complementary medicine.
\end{abstract}

Keywords: pine honey, oak honey, manuka, Brazilian propolis, synthetic antibiotic

Honey and propolis are important apitherapic agents, and they have many different biological activities, such as antimicrobial, antioxidant, anti-inflammatory, immune-modulator, antitumor, etc. (Ahuja \& Ahuja, 2010; CAn et al., 2015; Pobiega et al., 2019). Honey consists of carbohydrates (65-75\%), moisture (15-20\%), minerals, and various secondary metabolites (1-2\%) (CAN et al., 2015). The four main reasons explaining why honey is a good antimicrobial agent are: its $\mathrm{pH}$, viscosity, hydrogen peroxide source from glucose oxidase, and secondary metabolites (KoLAYLI et al., 2016). Except secondary metabolites, the other three substances are common in all honey samples. The amount, variety, and kind of the secondary metabolite differ according to honey types (AHuja \& AHuja, 2010). Raw propolis is composed mainly of resin $(40-50 \%)$, wax $(25-30 \%)$, essential compounds $(5-10 \%)$, pollens $(2-5 \%)$, and numerous other organic molecules (polyphenols, vitamins, and sugars) (KESKIN \& KoLAYLI, 2018). It was noted that propolis is one of the best pharmaceutical agents, and it contains many different bioactive compounds. The number of flavonoids and its phenyl esters were present in the extracts with antibacterial effects on pathological microorganisms. In this study, antimicrobial and antifungal effects of 42 different honey and eight propolis samples were compared.

\footnotetext{
* To whom correspondence should be addressed.

Phone: +902282141641; e-mail: merveozdemirkeskin@gmail.com
} 


\section{Materials and methods}

\subsection{Samples collections and test microorganisms}

In this study, 42 different honey samples were investigated. Honey samples were mostly collected from Turkey in 2016-2017 harvest seasons, and some of them were obtained from different countries as shown in Table 1. Four different grades of Unique Manuka Factor (UMF) certificated Manuka honey samples (UMF-10+, UMF12+, UMF15+, and UMF20+) were purchased from The Real Honey Company, England. Propolis samples were collected from different regions of Turkey. Brazilian Red propolis (raw) was purchased from a Brazilian company, Natura Nectar. All test microorganisms were obtained from the Hifzissihha Institute of Refik Saydam (Ankara, Turkey). Thirteen bacterial strains and 3 fungal strains (Ec: Escherichia coli ATCC 25922; Yp: Yersinia pseudotuberculosis ATCC 911; Kp: Klebsiella pneumonia subp. pneumonia ATCC18883; Pa: Pseudomonas aeruginosa ATCC 27853; Hp: Helicobacter pyloriii J99; Sa: Staphylococcus aureus ATCC 25923; Ef: Enterococcus faecalis ATCC 29212; Sm: Streptococcus mutans RSKK07038; Sag: Streptococcus agalactiae (clinic strain); Bc: Bacillus cereus 702 Roma; La: Lactobacillus acidophilus RSKK06029; Lc: Lactobacillus casei RSKK591; Ms: Mycobacterium smegmatis ATCC607; Ca: Candida albicans ATCC 60193; Ct: Candida tropicalis ATCC 13803; Sc: Saccharomyces cerevisiae) used in the current study were clinical isolates obtained from RTE University's Hospitals, Rize.

\subsection{Honey classifications, propolis extraction, and determination of total phenolic content}

The honey and propolis samples were obtained from different regions that have different botanical origin (Table 1). The honey samples were classified according to SANTIAGO and co-workers (2018). The propolis extracts were prapered according to KeSKIN and KoLAYLI (2018). Total phenolic compounds of the samples were determined using the Folin-Ciocalteu spectrophotometric assay (SinGLETon et al., 1999).

\subsection{Agar well diffusion method}

Simple susceptibility screening method was used by employing the agar-well diffusion method (Woods et al., 2003).

\subsection{Statistical analysis}

The analyses were performed three times, the results were presented as mean values and standard deviations. Regression analysis of the data was performed in Microsoft Office Excel 2013 (Microsoft Corporation, Redmond, WA, USA). 
Table 1. Specifications of studied honey and propolis samples

\begin{tabular}{|c|c|c|c|c|c|}
\hline $\begin{array}{l}\text { Sample } \\
\text { name }\end{array}$ & $\begin{array}{l}\text { Sample } \\
\text { code }\end{array}$ & Sample types & Region & Dominant pollens & Properties \\
\hline \multirow[t]{4}{*}{ Manuka } & $\mathrm{H} 1$ & Manuka UMF +10 & New Zealand & L. scoparium & Commercial \\
\hline & $\mathrm{H} 2$ & Manuka UMF +12 & “ & L. scoparium & Commercial \\
\hline & $\mathrm{H} 3$ & Manuka UMF +15 & “ & L. scoparium & Commercial \\
\hline & $\mathrm{H} 4$ & Manuka UMF +20 & “ & L. scoparium & Commercial \\
\hline \multirow{27}{*}{$\begin{array}{l}\text { Unifloral } \\
\text { honeys }\end{array}$} & H5 & Sunflower & Kırklareli/ & Helianthus annuus & Turkey \\
\hline & H6 & Sunflower & Tekirdag & Helianthus annuus & “ \\
\hline & $\mathrm{H} 7$ & Chestnut & Ordu & Castanea sativa & “ \\
\hline & H8 & Chestnut & Kure & Castanea sativa & “ \\
\hline & H9 & Astragalus & Palandöken & Astragalus microcephalus & “ \\
\hline & $\mathrm{H} 10$ & Astragalus & Erzurum & Astragalus microcephalus & “ \\
\hline & H11 & Thyme & Çanakkale & Thymus vulgaris & “ \\
\hline & $\mathrm{H} 12$ & R.caucasium & Rize & Rhododendron & “ \\
\hline & H13 & R.ponticum & Trabzon & Rhododendron & “ \\
\hline & H14 & Pumpkin & Izmir & Pumpkin & “ \\
\hline & H15 & Cultivated Thyme & Denizli & Thymus vulgaris & “ \\
\hline & H16 & Natural Thyme & Denizli & Thymus vulgaris & “ \\
\hline & H17 & Calltrop & Bursa & Eryngium campestre & “ \\
\hline & H18 & Thistle & Hatay & Silybium marianum & “ \\
\hline & H19 & Coriander & Burdur & Coriandrum sativum & “ \\
\hline & $\mathrm{H} 20$ & Harnup & Hatay & Ceratonia siliqua & “ \\
\hline & $\mathrm{H} 21$ & Black Cumin & Adana & Nigella sativa & “ \\
\hline & $\mathrm{H} 22$ & Nettleorurtica & Uskup & Urtica dioica & Macadonia \\
\hline & $\mathrm{H} 23$ & Heather & Mugla & Calluna vulgaris & Turkey \\
\hline & $\mathrm{H} 24$ & Heather & Mugla & Calluna vulgaris & “ \\
\hline & $\mathrm{H} 25$ & Buckwheat & Konya & Fagopyrum esculentum & “" \\
\hline & $\mathrm{H} 26$ & Buckwheat & Samsun & Fagopyrum esculentum & “ \\
\hline & $\mathrm{H} 27$ & Gorse & Kirklareli & Paliurus aculeatus & “ \\
\hline & $\mathrm{H} 28$ & Cedar & Hail & Cedrus ssp. & Saudi Arabia \\
\hline & $\mathrm{H} 29$ & AcaciaThomtree & Taif & Acacia ssp. & “ \\
\hline & $\mathrm{H} 30$ & Talha & Thomtree & Talha tree & “ \\
\hline & $\mathrm{H} 31$ & Ivy, Hedera & Kirklareli & Hedera helix & Turkey \\
\hline \multirow{7}{*}{$\begin{array}{l}\text { Honey } \\
\text { dew }\end{array}$} & H32 & Honeydew & Rize & Forest honey & Turkey \\
\hline & H33 & Honeydew & Gümüşhane & Forest honey & “ \\
\hline & H34 & Honeydew & Arsin & Forest honey & “ \\
\hline & H35 & Oak & Kirklareli & Oak spp. & “ \\
\hline & H36 & Oak & Samsun & Oak spp. & “ \\
\hline & H37 & Pine & Muğla & Pinus L. & “ \\
\hline & $\mathrm{H} 38$ & Pine & Izmir & Pinus L. & “ \\
\hline \multirow{4}{*}{$\begin{array}{l}\text { Multi- } \\
\text { floral }\end{array}$} & H39 & Blossom & Anzer & Plateau honey & “" \\
\hline & $\mathrm{H} 40$ & Blossom & Gümüşhane & Plateau honey & “ \\
\hline & H41 & Blossom & Hakkari & Plateau honey & “ \\
\hline & $\mathrm{H} 42$ & Blossom & Hakkari & Plateau honey & “ \\
\hline Raw & P1 & Red Brazilian & Brezillia & & Brazilia \\
\hline \multirow[t]{7}{*}{ Propolis } & P2 & Kars & Turkey & & Turkey \\
\hline & P3 & Yığılca & Turkey & & “ \\
\hline & P4 & Zonguldak & Turkey & & “ \\
\hline & P5 & Ankara & Turkey & & “ \\
\hline & P6 & Erzurum & Turkey & & “ \\
\hline & P7 & Konya & Turkey & & “ \\
\hline & P8 & Artvin & Turkey & & “ \\
\hline
\end{tabular}




\section{Results and discussion}

Total phenolic content of honey and propolis samples depends on geographical origin (KesKIN et al., 2020). In a study, it is reported that total amount of phenolic content of Anatolian raw propolis varies between 16.13-178.34 mg GA/g (KeSKIN \& KOLAYLI, 2018) and total amount of phenolic content of honey samples ranged between $33 \mathrm{mg} \mathrm{GA} / 100 \mathrm{~g}$ and $81 \mathrm{mg} \mathrm{GA} / 100 \mathrm{~g}$ (KeSKIN et al., 2020). It is clear from the obtained results that the unifloral and honeydew honey samples had higher phenolic compounds than multifloral honeys (Table 2). Although the honey samples showed different inhibition effects against the 16 microorganisms, the honey samples mostly affected E. coli, Y. pseudotuberculosis, K. pneumonia, S. aureus, and M. smegmatis (Table 3). P. aeruginosa, S. mutans, L. casei, and yeast like fungus of $C$. albicans, $C$. tropicalis, and $S$. cerevisiae were not affected by any of the honey samples. At the beginning of the study, Manuka honeys were used as positive controls, because numerous investigations in the literature show that these honeys have high antimicrobial activities. Surprisingly, only 4 microorganisms, Y. pseudotuberculosis, K. pneumonia, $S$. aureus, and M. smegmatis, were inhibited by the Manuka honey samples. Although Manuka UMF +10 and +12 samples had moderate antimicrobial effects on $H$. pylorii ( 8 and $10 \mathrm{~mm}$, respectively), heather honey from Muğla region had better activity against these bacteria $(12-15 \mathrm{~mm})$. Moreover, there were no substantial antimicrobial differences among the four Manuka honeys. Among the honey samples, H11-15, H17-19, $\mathrm{H} 21-23, \mathrm{H} 25-26, \mathrm{H} 31-32$, and H34-36 showed the highest inhibitions against $S$. aureus (Table 3). Although honey samples generally showed inhibition effects against $M$. smegmatis, cedar, acacia, and Talha (H28, H29, and H30) honey samples obtained from Saudi Arabia were the most effective honey samples against this microorganism. The three unifloral honeys of cedar (H28), acacia (H29), and Talha (H41) were found to be very effective especially against Lactobacillus acidophilus, L. casei, and M. smegmatis. Some bacteria (L. acidophilus, L. casei, and S. aureus) are related to dental health and tooth decay (YADAV \& PraKash, 2017), and the inhibition of these bacteria by honeys is an important finding. In general, there were no major differences found between the honey samples against the four bacteria ( $Y$. pseudotuberculosis, K. pneumonia, S. aureus, and M. smegmatis). Different authenticities of the honeys have also showed dissimilar inhibitions among the 16 microorganisms (Table 3).

For example, only Arabian honeys (H28, H29, and H30) and multifloral honey from Hakkari (H41) showed moderate inhibition against $S$. mutans. In addition, only two buckwheat honeys showed moderate inhibition against $C$. albicans and C. tropicalis. At the same time, only the buckwheat honeys and the oak honeys showed moderate inhibition against $S$. cerevisiae. Nearly half of the honey samples showed a weak inhibition against $L$. acidophilus, while the S. Arabic region honeys showed high inhibition effects. Saudi Arabian honeys had the highest phenolic contents (Table 2), and oak, chestnut, heather, buckwheat, and Manuka honeys had higher total phenolic contents than multifloral and blossom honeys. It was reported earlier that oak, chestnut, and heather honeys were dark colored honeys and contained higher phenolic compounds (CAN et al., 2015). Cedar, black cumin (Nigella sativa), and Manuka honeys showed a good bactericidal-bacteriostatic inhibition effect against only Staphylococcus aureus (AlMASAUDI et al., 2017), and our results supported these findings. Antimicrobial activity of honey samples could be due to the quantity and synergistic effect of key phenolics (KALOGEROPOULOS et al., 2009). The antimicrobial activities of the propolis extracts are given in Table 3. All propolis samples showed inhibition against the studied microorganisms to diferent extent, but the widest inhibition zone was found againts $H$. pylori, 


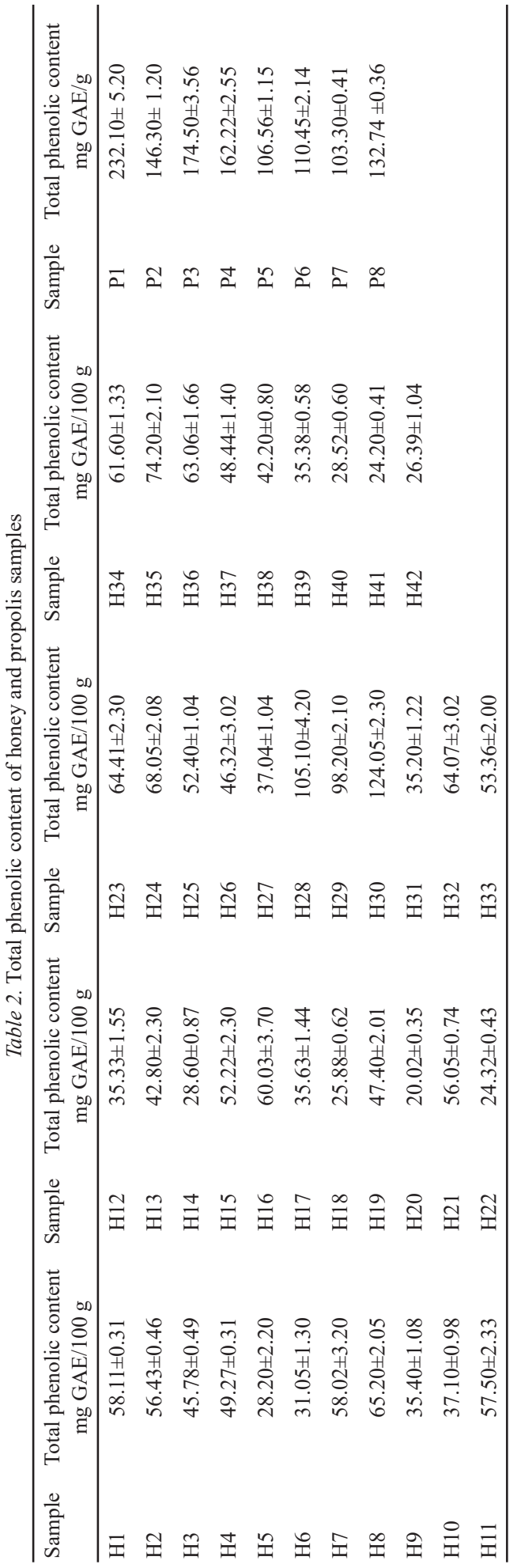


which is a fastidious, Gram negative bacterium that grows poorly in broth culture. Our findings showed that propolis extracts have much better inhibition effects than honey samples, which clearly shows that propolis is a much better antimicrobial agent than honey. All samples had the highest antimicrobial activity against $H$. pylori, with Yıgllca (P3) propolis showing the best results. In a previous study, gastric system bacteria were found sensitive to many different Anatolia propolis samples, the inhibition zone diameters ranged from 18 to 22 $\mathrm{mm}$ (Velikova et al., 2000). Moreover, in the same study, the anti-urease activity of Anatolia propolis was studied, and the ethanolic extracts showed a good inhibition of the extracellular urease of the bacteria. It was reported that these bee products, either honey or propolis, killed bacteria by inhibition of their urease enzyme (BALTAS et al., 2016). It was notably seen that all studied propolis samples showed good antimicrobial activity against Gram negative bacteria. In the previous studies, poplar type propolis samples were found ineffective and Bulgarian type was effective against E.coli (VeLIKova et al., 2000). The good activity found in this study can be due to similar constituents found in Bulgarian and Turkish propolis (Velikova et al., 2000). In this study, the highest total phenolic content in propolis was found in the Brazilian sample, showing a good inhibition against all bacteria and fungi to different extent. Some bacteria are even affected by low doses of propolis, while others need high doses. These findings are also confirmed by other studies (NETO et al., 2017). The propolis samples were also found very effective against oral pathogens such as Streptococcus mutans, Enterococcus faecalis, and C. albicans. Propolis samples have higher antimicrobial activity than honey samples, and the antimicrobial activity of propolis samples depend on their total phenolic content. Therefore, according to typification approach in the standardisation process, similar plant sources should be investigated for Brazilian and Turkish propolis to determine key chemicals providing the antimicrobial effect.

Table 3. Antimicrobial activities of honey samples against a range of microorganisms

\begin{tabular}{|c|c|c|c|c|c|c|c|c|c|c|c|c|c|c|c|c|c|}
\hline \multirow[t]{3}{*}{ Samples } & \multirow[t]{3}{*}{ Code } & \multicolumn{16}{|c|}{ Tested microorganisms and minimum inhibition zone diameters (mm) } \\
\hline & & \multicolumn{5}{|c|}{ Gram negative bacteria } & \multicolumn{7}{|c|}{ Gram positive bacteria } & \multirow{2}{*}{$\begin{array}{l}\text { Other } \\
\text { Ms }\end{array}$} & \multicolumn{3}{|c|}{ Yeast like fungi } \\
\hline & & Ec & Yp & Kp & $\mathrm{Pa}$ & Hp & $\mathrm{Sa}$ & Ef & $\mathrm{Sm}$ & Sag & $\mathrm{Bc}$ & $\mathrm{La}$ & $\mathrm{Lc}$ & & $\mathrm{Ca}$ & $\mathrm{Ct}$ & $\mathrm{Sc}$ \\
\hline \multirow[t]{4}{*}{ Manuka } & $\mathrm{H} 1$ & - & 8 & 6 & - & 8 & 8 & - & - & - & - & - & - & 6 & - & - & - \\
\hline & $\mathrm{H} 2$ & - & 6 & 6 & - & 10 & 8 & - & - & - & - & - & - & 6 & - & - & - \\
\hline & $\mathrm{H} 3$ & - & 6 & 6 & - & - & 8 & - & - & - & - & - & - & 6 & - & - & - \\
\hline & $\mathrm{H} 4$ & - & 8 & 8 & - & - & 8 & - & - & - & - & - & - & 8 & - & - & - \\
\hline \multirow{11}{*}{$\begin{array}{l}\text { Unifloral } \\
\text { honeys }\end{array}$} & H5 & - & 8 & 8 & - & - & 8 & - & - & - & 6 & 6 & - & 8 & - & - & - \\
\hline & H6 & - & 6 & 6 & - & - & 10 & - & - & - & 6 & 6 & - & 8 & - & - & - \\
\hline & H7 & 8 & 8 & 6 & - & - & 10 & - & - & - & - & 6 & - & 10 & - & - & - \\
\hline & H8 & 8 & 10 & 8 & - & - & 10 & - & - & - & - & - & - & 10 & - & - & - \\
\hline & H9 & 6 & 10 & 8 & - & - & 10 & 6 & - & - & - & - & - & 6 & - & - & - \\
\hline & $\mathrm{H} 10$ & 6 & 10 & 8 & - & - & 10 & 6 & - & - & - & - & - & 6 & - & - & - \\
\hline & H11 & - & 6 & 8 & - & - & 14 & - & - & - & - & 6 & - & 6 & - & - & - \\
\hline & H12 & 6 & 6 & 8 & - & - & 15 & - & - & - & - & 6 & - & - & - & - & - \\
\hline & H13 & 8 & 6 & 8 & - & - & 16 & 6 & - & - & - & 6 & - & - & - & - & - \\
\hline & H14 & - & 6 & - & - & - & 15 & - & - & - & - & - & - & 6 & - & - & - \\
\hline & H15 & - & 6 & 8 & - & 10 & 14 & - & - & - & 10 & 8 & - & 6 & - & - & - \\
\hline
\end{tabular}


Table 3. cont

\begin{tabular}{|c|c|c|c|c|c|c|c|c|c|c|c|c|c|c|c|c|c|}
\hline \multirow[t]{3}{*}{ Samples } & \multirow[t]{3}{*}{ Code } & \multicolumn{16}{|c|}{ Tested microorganisms and minimum inhibition zone diameters (mm) } \\
\hline & & \multicolumn{5}{|c|}{ Gram negative bacteria } & \multicolumn{7}{|c|}{ Gram positive bacteria } & \multirow{2}{*}{$\begin{array}{l}\text { Other } \\
\text { Ms }\end{array}$} & \multicolumn{3}{|c|}{ Yeast like fungi } \\
\hline & & Ec & Yp & $\mathrm{Kp}$ & $\mathrm{Pa}$ & Hр & $\mathrm{Sa}$ & Ef & $\mathrm{Sm}$ & Sag & $\mathrm{Bc}$ & $\mathrm{La}$ & Lc & & $\mathrm{Ca}$ & $\mathrm{Ct}$ & $\mathrm{Sc}$ \\
\hline \multirow{16}{*}{$\begin{array}{l}\text { Unifloral } \\
\text { honeys }\end{array}$} & H16 & - & 6 & 8 & - & 8 & 12 & - & - & - & 8 & 6 & - & 8 & - & - & - \\
\hline & H17 & - & 6 & 8 & - & - & 16 & 12 & - & - & 12 & 12 & - & 6 & - & - & - \\
\hline & H18 & - & 6 & 10 & - & - & 16 & - & - & - & - & 6 & - & 6 & - & - & - \\
\hline & H19 & 6 & 6 & 8 & - & - & 15 & - & - & - & 6 & 8 & - & 8 & - & - & - \\
\hline & $\mathrm{H} 20$ & - & 6 & - & - & - & 6 & - & - & - & - & - & - & 6 & - & - & - \\
\hline & $\mathrm{H} 21$ & - & 8 & 10 & - & - & 18 & - & - & - & 6 & 6 & - & 10 & - & - & - \\
\hline & $\mathrm{H} 22$ & - & 6 & 6 & - & - & 16 & - & - & - & - & - & - & 6 & - & - & - \\
\hline & $\mathrm{H} 23$ & 8 & 6 & 6 & - & 15 & 18 & - & - & - & - & - & - & 6 & - & - & - \\
\hline & $\mathrm{H} 24$ & - & 10 & - & & 12 & 10 & - & - & - & - & - & - & 11 & - & - & - \\
\hline & $\mathrm{H} 25$ & 8 & 8 & 10 & - & 8 & 20 & - & - & - & - & - & - & 10 & 12 & 10 & 12 \\
\hline & $\mathrm{H} 26$ & 10 & 10 & 10 & - & 10 & 16 & - & & & - & - & - & 10 & 10 & 10 & 10 \\
\hline & $\mathrm{H} 27$ & - & 6 & 6 & - & - & 12 & - & - & - & - & 8 & - & 6 & - & - & - \\
\hline & H 28 & 6 & 10 & - & - & 8 & 10 & - & 8 & - & - & 15 & 15 & 15 & - & - & - \\
\hline & $\mathrm{H} 29$ & 8 & 12 & 6 & 10 & 10 & 10 & - & 8 & - & - & 20 & 30 & 30 & - & - & - \\
\hline & H30 & 8 & 10 & 10 & 10 & 10 & 11 & - & 8 & - & - & 20 & 30 & 15 & - & - & - \\
\hline & H31 & 8 & 12 & 8 & - & - & 14 & - & & - & - & 14 & 10 & 14 & - & - & - \\
\hline \multirow[t]{7}{*}{ Honeydew } & H32 & 10 & 10 & 10 & - & - & 16 & 6 & - & - & - & 10 & - & 12 & - & - & - \\
\hline & H33 & 8 & 8 & 8 & - & 8 & 10 & - & - & - & - & 10 & - & 10 & - & - & - \\
\hline & H34 & - & 6 & 7 & - & 10 & 16 & 6 & - & - & 6 & 10 & - & 10 & - & - & - \\
\hline & H35 & 12 & 16 & 8 & 8 & 10 & 15 & 6 & - & - & 8 & 10 & - & 8 & - & - & 10 \\
\hline & $\mathrm{H} 36$ & 10 & 14 & 6 & 8 & 8 & 18 & - & - & - & 8 & - & - & 8 & - & - & 8 \\
\hline & H37 & - & 12 & 6 & 8 & - & 12 & - & - & - & - & - & - & 8 & - & - & - \\
\hline & $\mathrm{H} 38$ & - & 10 & 6 & 8 & - & 10 & - & - & - & - & - & - & 8 & - & - & - \\
\hline \multirow[t]{4}{*}{ Mulifloral } & H39 & 8 & 8 & 6 & 8 & - & 10 & - & - & - & - & - & - & 8 & - & - & - \\
\hline & $\mathrm{H} 40$ & - & - & 10 & 6 & 10 & 10 & 10 & - & - & - & - & - & 6 & - & - & - \\
\hline & H41 & - & 6 & 6 & 6 & 8 & 10 & 15 & 8 & - & - & 8 & 6 & 6 & 6 & - & - \\
\hline & $\mathrm{H} 42$ & - & - & - & 6 & 8 & 10 & - & - & - & - & - & - & 6 & - & - & - \\
\hline
\end{tabular}

Ec: Escherichia coli ATCC 25922, Yp: Yersinia pseudotuberculosis ATCC 911, Kp: Klebsiella pneumonia subsp. pneumonia ATCC18883, Pa: Pseudomonas aeruginosa ATCC 27853, Hp: Helicobacter pylorii J99, Sa: Staphylococcus aureus ATCC 25923, Ef: Enterococcus faecalis ATCC 29212, Sm: Streptococcus mutans RSKK07038, Sag: Streptococcus agalactiae (clinical strain), Bc: Bacillus cereus 702 Roma, La: Lactobacillus acidophilus RSKK06029, Lc: Lactobacillus casei RSKK591, Ms: Mycobacterium smegmatis ATCC607, Ca: Candida albicans ATCC 60193, Ct: C. tropicalis ATCC 13803, Sc: Saccharomyces cerevisiae RSKK 251, (-): No activity. 6-9 mm; low activity, 9-11 mm; moderate activity, $\geq 12$; good activity 
Table 4. Antimicrobial activities of the ethanolic propolis samples against a range of microorganisms

\begin{tabular}{|c|c|c|c|c|c|c|c|c|c|c|c|c|c|c|c|c|}
\hline \multirow{3}{*}{$\begin{array}{l}\text { Propolis } \\
\text { samples }\end{array}$} & \multicolumn{16}{|c|}{ Tested microorganisms and inhibition zone diameters $(\mathrm{mm})$} \\
\hline & \multicolumn{5}{|c|}{ Gram negative bacteria } & \multicolumn{7}{|c|}{ Gram positive bacteria } & \multirow{2}{*}{$\begin{array}{c}\text { Other } \\
\text { Ms }\end{array}$} & \multicolumn{3}{|c|}{ Yeast like fungi } \\
\hline & Ec & Yp & $\mathrm{Kp}$ & $\mathrm{Pa}$ & $\mathrm{Hp}$ & $\mathrm{Sa}$ & Ef & Sm & Sag & $\mathrm{Bc}$ & $\mathrm{La}$ & $\mathrm{Lc}$ & & $\mathrm{Ca}$ & $\mathrm{Ct}$ & Sc \\
\hline P1 & 8 & 15 & 11 & 12 & 45 & 22 & 20 & 12 & 12 & 18 & 24 & 12 & 20 & 16 & 14 & 20 \\
\hline P2 & 8 & 10 & 8 & 24 & 40 & 18 & 8 & 6 & 6 & 12 & 14 & 6 & 15 & 14 & 6 & 8 \\
\hline P3 & - & 10 & 8 & 12 & 50 & 20 & 12 & 10 & 10 & 14 & 25 & 12 & 18 & 12 & 12 & 20 \\
\hline P4 & 12 & 10 & 10 & 18 & 45 & 20 & 15 & 12 & 12 & 12 & 22 & 14 & 25 & 14 & 12 & - \\
\hline P5 & 8 & 6 & 14 & 8 & 40 & 10 & 15 & 6 & 6 & 15 & 15 & 6 & 18 & 6 & 6 & 10 \\
\hline P6 & 10 & 10 & 12 & 10 & 45 & 14 & 10 & 6 & 6 & 14 & 18 & 8 & 17 & 15 & 8 & 15 \\
\hline P7 & 12 & 10 & 6 & 10 & 40 & 16 & 15 & 10 & 10 & 14 & 18 & 10 & 15 & 15 & 12 & 14 \\
\hline P8 & 8 & 8 & 6 & 18 & 40 & 16 & 10 & 10 & 8 & 8 & 18 & 10 & 12 & 10 & 8 & - \\
\hline Amp. & 10 & 10 & 10 & 18 & NT & 35 & 10 & NT & NT & 15 & NT & NT & & & & \\
\hline Strep. & & & & & & & & & & & & & 35 & & & \\
\hline Flu. & & & & & & & & & & & & & & 25 & 25 & 25 \\
\hline
\end{tabular}

Ec: Escherichia coli ATCC 25922; Yp: Yersinia pseudotuberculosis ATCC 911; Kp: Klebsiella pneumonia subsp. pneumonia ATCC18883; Pa: Pseudomonas aeruginosa ATCC 27853; Hp: Helicobacter pyloriii J99; Sa: Staphylococcus aureus ATCC 25923; Ef: Enterococcus faecalis ATCC 29212; Sm: Streptococcus mutans RSKK07038; Sag: Streptococcus agalactiae (clinical strain); Bc: Bacillus cereus 702 Roma; La: Lactobacillus acidophilus RSKK06029; Lc: Lactobacillus casei RSKK591; Ms: Mycobacterium smegmatis ATCC607; Ca: Candida albicans ATCC 60193; Ct: C. tropicalis ATCC 13803; Sc: Saccharomyces cerevisiae RSKK 251; (-): No activity. 6-9 mm; low activity; 9-11 mm; moderate activity; $\geq 12$; good activity

\section{Conclusions}

Honey and propolis are substantial antibacterial and antifungal agents, and their antimicrobial effects could result from their floral sources, but antimicrobial activities were found not to be dependent on their total phenolic contents. For this reason, further studies are needed to evaluate those mechanisms. Better antimicrobial effects of propolis implied that wherever they live, bees are created to sense, find, and collect the best chemicals in any environment to protect their hives against microorganisms. Therefore, this natural product could be used in preservative and complementary medicine.

Funding: This study was supported by TUBITAK [grant number 114Z370].

\section{References}

Ahuja, A. \& Ahuja, V. (2010): Apitherapy - A sweet approach to dental diseases. Part I: Honey. J. Adv. Dental Res., $1,81-86$.

Almasaudi, S.B., Al Nahari, A.A., El Sayed, M., Barbour, E., Al Muhayawi, S. M., ... \& Harakeh, S. (2017): Antimicrobial effect of different types of honey on Staphylococcus aureus. Saudi J. Biol. Sci., 24(6), 12551261.

Baltas, N., Yildiz, O. \& KolaYli, S. (2016): Inhibition properties of propolis extracts to some clinically important enzymes. J. Enzyme Inhib. Med. Ch., 31, 52-55.

Acta Alimentaria 49, 2020 
Can, Z., Yildiz, O., Şahin, H., Asadov, A. \& Kolayli, S. (2015): Phenolic profile and antioxidant potential of propolis from Azerbaijan. Mellifera, 15(1), 16-28.

Kalogeropoulos, N., Konteles, S.J., Troullidou, E., Mourtzinos, I.\& Karathanos, V.T. (2009): Chemical composition, antioxidant activity and antimicrobial properties of propolis extracts from Greece and Cyprus. Food Chem., 116(2), 452-461.

Keskin, M. \& Kolayli, S. (2018): Standardization of propolis, is it possible? Uludag Bee J., 18(2),101-110.

KESKIN, Ş., MAYDA, N., KESKIN, M. \& ÖZKÖK, A. (2020): Investigation of Bilecik honeys in terms of melissopalynology and chemical analyses. GIDA -J. Food, 45(2), 275-289.

Kolayli, S., CakiR, H.E. \& Sahin, H. (2016): Anti-inflammatory activities of some bee products by inhibition of bovine testes hyaluronidase. Curr. Enzyme Inhib., 12(2), 183-187.

Neto, M.R., Tintino, S.R., Da Silva, A.R.P., Do Socorro Costa, M., Boligon, A.A., ... \& Coutinho, H.D.M. (2017): Seasonal variation of Brazilian red propolis: Antibacterial activity, synergistic effect and phytochemical screening. Food Chem. Toxicol., 107, 572-580

PobieGa, K., KraŚNIEWSKA, K. \& GNiEwosz, M. (2019): Application of propolis in antimicrobial and antioxidative protection of food quality - A review. Trends Food Sci. Tech., 83, 53-62.

Santiago, K.B., Piana, G.M., Conti, B.J., Cardoso, E.D.O., Murbach Teles Andrade, B. F., ... \& Sforcin, J.M. (2018): Microbiological control and antibacterial action of a propolis-containing mouthwash and control of dental plaque in humans. Nat. Prod. Res., 32(12), 1441-1445.

Singleton, V.L., Orthofer, R. \& LamUela-RaVEntós, R.M. (1999): Analysis of total phenols and other oxidation substrates and antioxidants by means of Folin-Ciocalteu reagent. Method. Enzymol., 299, 152-178.

Velikova, M., Bankova, V., Sorkun, K., Houcine, S., Tsvetkova, I. \& Kujumgiev, A. (2000): Propolis from the Mediterranean region: chemical composition and antimicrobial activity. Z. Naturforsch. C., 55(9,10), 790793.

Woods, G.L., Brown-Elliott, B., Desmond, E.P., Hall, G.S., Heifets, L., Pfyffer, G.E. \& Witebsky, F.G. (2003): Susceptibility testing of mycobacteria, nocardiae, and other aerobic actinomycetes; Approved standard. CLSI publication / Clinical and Laboratory Standards Institute, M24-A2.

Yadav, K. \& Prakash, S. (2017): Dental caries: A microbiological approach. J. Clin. Infect Dis. Pract., 2(1), 1-15.

Open Access statement. This is an open-access article distributed under the terms of the Creative Commons Attribution-NonCommercial 4.0 International License (https://creativecommons.org/licenses/by-nc/4.0/), which permits unrestricted use, distribution, and reproduction in any medium for non-commercial purposes, provided the original author and source are credited, a link to the CC License is provided, and changes - if any - are indicated. 